\title{
A Framework for Pedagogical Feedback in the Motor Skill Domain
}

\author{
Yulita Hanum P Iskandar, Lester Gilbert, Gary B Wills \\ Learning Societies Lab, \\ School of Electronics and Computer Sciences, University of Southampton, \\ Southampton, SO17 IBJ, UK. \\ \{yhpi07r,lg3,gbw\}@ecs.soton.ac.uk
}

\begin{abstract}
With increasingly rapid development in Computerbased Sport Training (CBST), feedback plays an important role in both coaching and learning. A good CBST system includes not only good training strategies but also effective feedback design. Feedback in the motor skill domain via CBST may be synthetically designed to allow athletes to practise in a more effective way, and enhance their skill acquisition. Existing designs lack pedagogical elements. To bridge the gap, we propose a framework for the design of pedagogically-informed feedback based on learning transactions, competence, cybernetics, and behaviourism.
\end{abstract}

\section{Introduction}

Motor skills, although not usually the major part of educational objectives in Higher Education, are components of a distinct type of learning outcome and essential to learning and teaching in human performance. Objectives in skilled performance are different from cognitive objectives which typically involve declarative, procedural, or conditional knowledge. Well-executed motor skills are precise, smooth, continuous, and accurately timed performances characteristically associated with sport.

The development of Computer-based Sport Training (CBST) has made it possible to augment and improve the feedback that athletes receive during training. Feedback systems incorporate embedded sensors and devices into the sports equipment and use sensors attached to the athlete to acquire information about learning processes and the achievement of intended outcomes. Through feedback, athletes recognize areas of deficiency in their knowledge and skills which they seek to remedy.
This feedback can be intrinsic or extrinsic; the latter can consist of knowledge of results or knowledge of performance. Knowledge of results allows performers to examine their efforts in relation to an externally defined goal. However, such information feedback provides only goal-related information and ignores knowledge of performance, which is information about how the action was completed. Pedagogically designed feedback in the motor skill domain allows athletes to know the performance goal and perceive the need to carry out corrections relative to some expected training outcome. The pedagogically designed feedback allows adaptive training experiences that are tailored to the different needs and characteristics of the athletes, especially in terms of their current competence.

The structure of the paper as follows: Section 2 analyzes literature on CBST, Section 3 discusses related work, Section 4 proposes a framework for pedagogical feedback, and Section 5 presents our conclusion.

\section{Computer-based sport training}

Computer-based technology (e.g., virtual reality, motion training systems, and ergometer machines) has been introduced into the sport domain and is used to record athletes' performance whilst they interact with the system. Thus, CBST serves as both a stimulus towards and a method for the study of choices that athletes make during athlete-controlled training opportunities.

Instruction via CBST can readily incorporate provision for athletes to respond and for feedback appropriate to that response. In the practice of basic skill, CBST can react to the quickness of the athlete's response. In view of the desirability of attaining automaticity in certain basic skills, measuring athletes' speed of response to practice examples is likely to be very useful in performance assessment. Quickness of response is one indicator of skill automaticity, which is 
of great significance as a prerequisite for additional learning and problem-solving.

CBST has had a profound impact on sport and many athletes and coaches now consider information derived from technological advances to be invaluable. This might be related to the concept of feedback that originated in mechanical control theory. Feedback provided in well-designed computer instruction is precise in relation to the correctness or degree of correctness of the athlete's response. For this reason, CBST is well-suited to the learning of skill.

\section{Related work}

Effective feedback to athletes has been identified as a key strategy in motor skill learning. Effective feedback is associated with feedback that is both appropriate and timely suited to the needs of the situation, sufficient, and instructor delivered. Therefore, feedback in CBST contributes to learning by allowing athletes to verify their movements, evaluate their progress, and determine the cause of errors. It also motivates them to remain involved in the training tasks, given that they perceive the feedback as helpful. This requires the active processing of feedback which is specific as well as feedback which addresses general metacognitive knowledge and strategies.

Hence, there exists a large variety of information that might be provided as feedback. The challenge for educational researchers and designers of CBST environments is to determine what constitutes effective and appropriate feedback for athletes in their training trajectory.

Research has focused on feedback's role in the cognitive domain $[1,2]$, but little research has focused on designing and implementing feedback in the motor skill domain via CBST. Currently, issues of feedback in the motor skill domain via CBST concern:

1. delivery of the feedback contents such as speed, accuracy, movement, time, and reaction time [3, 4],

2. providing athletes with access to their feedback via an appropriate user interface [5], and

3. modality of feedback, such as visual, audio, tactile, and haptic feedback [6].

Feedback in the cognitive domain and in motor skill environments is designed to shape the perception, cognition, or action of the learner. However, the design of feedback in the motor skill domain via CBST is typically led by technology and fails to properly consider pedagogical issues. Feedback in CBST does not usually derive from the goals, actions, performances, outcomes and contexts of a learning process. Thus, for pedagogical reasons, this paper proposes the design of effective feedback that can:

1. support athletes in their achievement of the underlying intended learning outcomes,

2. assist athletes in identifying the gaps in their performance, and

3. help athletes to determine performance expectations, identify what they have already learned and what they need to learn next, and judge their personal learning progress.

\section{Framework for pedagogical feedback in the motor skill domain}

To provide a foundation of pedagogy for feedback, principles involved in learning transactions, competence, cybernetics, and behaviourism were included in a framework (see Figure 1). These principles were chosen as they repetitively surfaced in research as the keys to effective teaching and learning.

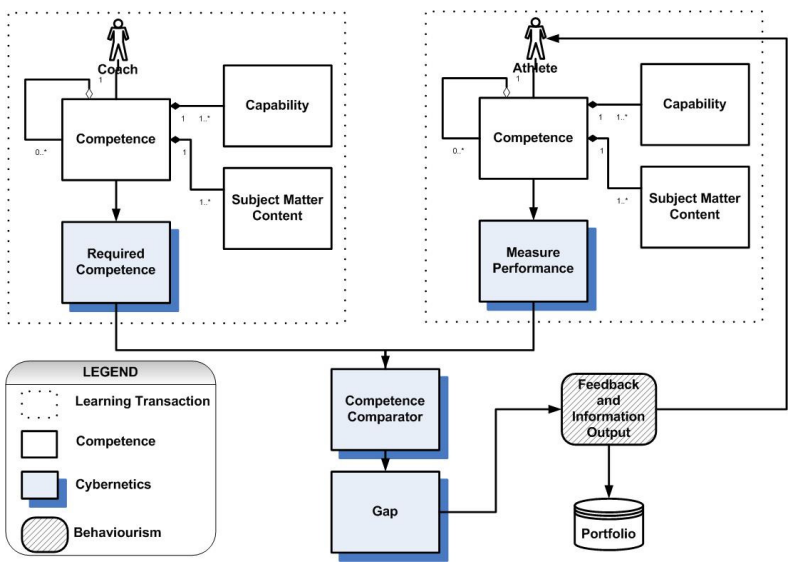

Figure 1: Framework of pedagogical feedback in the motor skill domain

\subsection{Learning transaction}

The learning transaction is a model (see Figure 2) of "what goes on" at the coach-athlete interface, providing an understanding of what is needed to analyze, design and implement pedagogically designed feedback in the motor skill domain [7]. It is anticipated that pedagogical feedback in this context can be straightforwardly designed and engineered, given an appropriate specification of these capabilities as they are required to be learned in a virtual rowing system. 


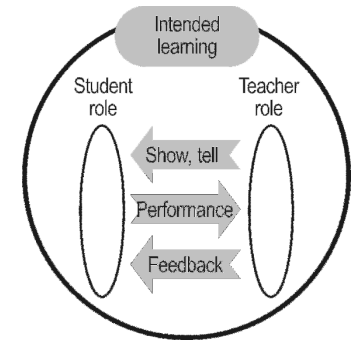

Figure 2: Learning transaction diagram

\subsection{Competence}

Competency can be defined as a measurable skill in reference to a given context. A competence may be conceptualised as a subject matter component, based upon knowledge representation models, and an action component (capability) which describes how the knowledge or subject matter is used (Figure 3). There are taxonomies which classify the action components, such as Dave's taxonomy [8]. The classified action components describe different motor skill processing modes and can be characterised with specific action verbs.

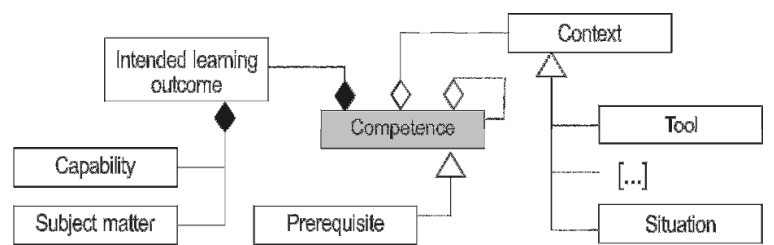

Figure 3: Competence conceptual model

\subsection{Cybernetics}

Cybernetics provides a model where discrepancies in performance capabilities can be identified and corrective action taken. The analysis of pedagogic feedback in the motor skill domain from a cybernetic point of view has four major components (1) measurement of the current competency of the athlete, (2) statement of the required standard of the competency, (3) comparison of the current competency to the required competency, and (4) corrective feedback and information. Hence, the competence comparator measures the performance of the athlete and compares it with the required competence as defined by the coach. The result is a gap analysis, which yields the required feedback and information output.

\subsection{Behaviourism}

Behaviourism was used as the basis of feedback and information output. From a behaviourist perspective, pedagogical feedback should be designed as a result of a task analysis.

\section{Conclusion}

In this paper, we proposed a framework of pedagogically designed feedback in the motor skill domain. The proposed feedback is design-oriented (focusing on means to attain given goals for learning or development), rather than description-oriented (focusing on the results of given events). The framework of pedagogical feedback in the motor skill domain draws a picture of how the principles from learning transaction, competency, cybernetics, and behaviourism work together to build sound pedagogical feedback for the implementation of a CBST system. We believe that effective pedagogical feedback in the motor skill domain is critical to successfully ensuring a pedagogic focus on coaching and learning activities in CBST.

\section{References}

1. Mory, E., Feedback research review. Handbook of research on educational communications and technology, 2004: p. 745-783.

2. Shute, V., Focus on Formative Feedback. Review of Educational Research, 2008. 78(1): p. 153.

3. Baudouin, A. and D. Hawkins, Investigation of biomechanical factors affecting rowing performance. Journal of Biomechanics, 2004. 37(7): p. 969-976.

4. Cheng, L. and S. Hailes, Managed exercise monitoring: a novel application of wireless on-body inertial sensing. 2008.

5. Cyboran, V., Designing feedback for computer-based training. Performance + Instruction, 1995. 34(5): p. 18-23.

6. Philo Tan, C., et al. Training for physical tasks in virtual environments: Tai Chi. in Virtual Reality, 2003. Proceedings. IEEE. 2003.

7. Gilbert, L. and V. Gale, Principles of Elearning Systems Engineering. 2008: Chandos.

8. Kennedy, D., Á. Hyland, and N. Ryan, Writing and using learning outcomes: a practical guide. University College Cork, 2007. 